\title{
HAX-1 inhibits apoptosis in prostate cancer through the suppression of caspase- 9 activation
}

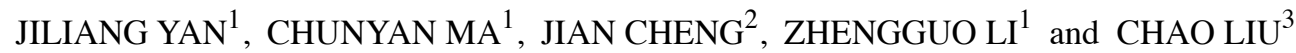 \\ ${ }^{1}$ Department of Clinical Laboratory, Kaifeng Central Hospital, Kaifeng, Henan 475000; \\ ${ }^{2}$ Department of Oncology, Taishan Medical University Affiliated Zouping Hospital, Zouping, Shandong 256200; \\ ${ }^{3}$ Department of Developmental Biology, Liaoning Medical University, Jinzhou, Liaoning 121001, P.R. China
}

Received June 10, 2015; Accepted July 20, 2015

DOI: $10.3892 / o r .2015 .4202$

\begin{abstract}
HS1 associated protein X-1 (HAX-1), a substrate of Src family tyrosine kinases, plays a critical role in cell apoptosis. However, its functions in prostate cancer remains unclear. The present study explored the role and mechanism of HAX-1 in cancer cell apoptosis. The mRNA and protein levels of $\mathrm{HAX}-1$ in the prostate cancer cell lines $\mathrm{PC}-3$, VCaP and DU145 were assessed. Cell proliferation, apoptosis and caspase-9 activities were assessed in DU145 after HAX-1 siRNA treatment. The mRNA and protein levels of HAX-1 in prostate cancer cell lines PC-3, VCaP and DU145 were significantly higher than those in the primary prostate epithelial cells, and DU145 possess the highest mRNA and protein levels compared to PC-3 and VCaP. When HAX-1 was knocked down in DU145, cell proliferation was significantly decreased, accompanied by a decrease in $\mathrm{Ki} 67$ protein expression. Compared with the control and control siRNA groups, HAX-1 siRNA promoted cell apoptosis and caspase- 9 activation in DU145. Furthermore, prostate cancer cells co-transfected with HAX-1 and caspase-9 promoted viability and reduced apoptosis. In contract, co-transfection of caspase-9 and HAX-1 siRNA suppressed the cell viability and enhanced apoptosis. In summary, the present study demonstrated that HAX-1 inhibits cell apoptosis through caspase-9 inactivation.
\end{abstract}

\section{Introduction}

Prostate cancer is one of the leading cause of cancer-related death in males, second only to lung cancer (1). Multiple genetic and epigenetic factors have been implicated in the oncogenesis and progression of prostate cancer. Prostate cancer initially responds well to androgen deprivation, but this treatment

Correspondence to: Dr Jiliang Yan, Department of Clinical Laboratory, Kaifeng Central Hospital, 85 Hedao Street, Kaifeng, Henan 475000, P.R. China

E-mail: liuqing_medical@163.com

Key words: HS1-associated protein X-1, caspase-9, apoptosis, prostate cancer results in the emergence of androgen-independent disease that is resistant to apoptosis (2).

HS1-associated protein X-1 (HAX-1) originally identified as a $35 \mathrm{kDa}$ protein that interacts with HS1, is a substrate of Src family tyrosine kinases (3). The $H A X-1$ gene is ubiquitously expressed among tissues (4), and its protein is localized mainly in mitochondria, and also in endoplasmic reticulum and nuclear envelope in the cells.

HAX-1 was suggested to be involved in the regulation of apoptosis (programmed cell death) (3). As an anti-apoptosis factor, the anti-apoptotic, cell-protecting properties of HAX-1 as well as its interactions with apoptosis-related proteins have been widely reported $(5,6)$. HAX-1 has been reported to protect the cultured cells against the challenge of $\mathrm{H}_{2} \mathrm{O}_{2}(7)$. $\mathrm{HAX}-1$ is highly expressed in colorectal cancer and contributes to the malignant progression and predict poor prognosis for patients with colorectal cancer (8). So far, the expression levels and the effect of HAX-1 in prostate cancer remain unclear.

Caspase-9, a protein also localized mainly in mitochondria is a key regulatory player in the mitochondria-mediated apoptosis pathway. Activated caspase- 9 directly cleaves and activates caspase- 3 and -7, resulting in the biochemical destruction of the cells (9). The inhibitor of apoptosis proteins (IAPs) have been thought to be the only class of proteins that directly inhibits caspase-9 as previously described (10), however, new mechanistic evidence showed that HAX-1 averts cell death by blocking the biological activation of caspase-9.

In the present study, we investigated the expression of HAX-1 in prostate cancer cell lines, PC3, VCaP and DU145. The role and the underlying mechanism of HAX-1 and caspase-9 interaction in prostate cancer cell apoptosis were also explored.

\section{Materials and methods}

Cell lines and cell culture. The primary human prostate epithelial cells and the human prostate cancer cell lines PC3, VCaP and DU145 were purchased from the American Type Culture Collection (ATCC; Manassas, VA, USA). The primary human prostate epithelial cells were cultured in Dulbecco's modified Eagle's medium (DMEM), and the human prostate cancer cell lines PC3, VCaP and DU145 were cultured in RPMI-1640 supplemented with $10 \%$ fetal bovine serum (FBS), $2 \mathrm{mmol} / \mathrm{l}$ 
GlutaMAX and $1 \%$ antibiotics (Invitrogen, Carlsbad, CA, USA) at $37^{\circ} \mathrm{C}$ in a humidified $5 \% \mathrm{CO}_{2}$ incubator. These cells were maintained in the appropriate medium and passaged every three days.

siRNA transfection. HAX-1 and control siRNAs were purchased from Santa Cruz Biotechnology (Santa Cruz, CA, USA). For transfection, $5 \times 10^{4}$ cells were seeded in each well of 24-well microplates and grown for $24 \mathrm{~h}$ to reach $60-65 \%$ confluency, and then incubated with a mixture of siRNA and Lipofectamine 2000 reagent (Invitrogen) in $100 \mu 1$ of serum-free Opti-MEM according to the manufacturer's instructions. The transfection efficiency was examined by real-time PCR and western blotting.

Construction of expression vectors and cell transfection. Total RNA from cells were isolated using TRIzol reagent (Invitrogen), and then converted to cDNA using the PrimeScript ${ }^{\circledR}$ RT reagent kit (Takara, Dalian, China) with oligo(dT) primers. Then, the open reading frame of caspase- 9 cDNA was cloned and inserted into the pcDNA3.1 vector (Invitrogen) to construct the recombinant pcDNA3.1-caspase-9 expression vector. Control and pcDNA3.1 vector transfected cells were also prepared. For cell transfection, cells were cultured to $60 \%$ confluency and transfection was performed using the FuGENE HD transfection reagent (Roche, Indianapolis, IN, USA) method as suggested by the manufacturer.

Real-time PCR (RT-PCR). Total RNA was isolated as previously described. Primers for HAX-1 were designed as: 5'-AAC CAGAGAGGACAATGATCT-3' (sense), and 5'-AAGTTGTCC AAAGAAACCTGT-3' (antisense). $\beta$-actin was used as the normalization control for HAX-1 and caspase- 9 gene. The $25 \mu 1$ reaction mixture contained $12.5 \mu 12 \mathrm{X}$ OneStep qRT-PCR buffer, $0.5 \mu \mathrm{M}$ reverse and $0.5 \mu \mathrm{M}$ forward primer, $0.9 \mu \mathrm{l}$ enzymix, $90 \mathrm{ng}$ RNA template and $0.5 \mu \mathrm{M}$ probe. PCR conditions for the reverse transcription used to obtain cDNA were as follows: $45^{\circ} \mathrm{C}$ for $10 \mathrm{~min}$, pre-denaturation at $95^{\circ} \mathrm{C}$ for $10 \mathrm{~min}$ and then 45 cycles of $95^{\circ} \mathrm{C}$ for $15 \mathrm{sec}$ and $60^{\circ} \mathrm{C}$ for $45 \mathrm{sec}$; this was performed using the ABI 7500 Real-Time PCR System (Applied Biosystems, Foster City, CA, USA). Real-time RT-PCR was performed in a Rotor-Gene RG-3000 Real-Time Thermal Cycler (Corbett Research, Sydney, Australia).

Western blotting. The proteins were extracted from cells using RIPA lysis buffer (Beyotime, Nantong, China). For western blotting, equal amounts of proteins were separated on SDS-PAGE and blotted onto a pre-wet nitrocellulose membrane (GE Healthcare, Germany), followed by blocking of membranes in $10 \%$ defatted milk in phosphate-buffered saline (PBS) at $4^{\circ} \mathrm{C}$ overnight, the membranes were then probed with different primary antibodies. The primary antibodies were as follows: mouse anti-HAX-1, mouse antiBcl-2, mouse anti-Bax and mouse anti- $\beta$-actin antibodies (Santa Cruz Biotechnology), rabbit anti-caspase-9 antibody (Abcam, Cambridge, UK). After washing with TBST buffer, the membranes were incubated for $1 \mathrm{~h}$ at $25^{\circ} \mathrm{C}$ with HRP-conjugated secondary antibodies. ECL reagent was used for detection. The fluorescence was scanned using a Typhoon scanner (Amersham Biosciences, Piscataway, NJ, USA). All experiments were performed in triplicate.

Cell viability. The cell viability measurements were carried out using the MTT assay. Approximately $5 \times 10^{4}$ cells were seeded into 96-well plates, washed twice with PBS and $10 \mu 1$ of MTT was added to each well. Then, the cells were incubated at $37^{\circ} \mathrm{C}$ for $2 \mathrm{~h}$ and $100 \mu \mathrm{l}$ dimethylsulfoxide (DMSO) was added to dissolve the formazan crystals. Absorbance was measured at $560 \mathrm{~nm}$ with a SpectraMax Paradigm Multi-Mode Reader (Molecular Devices, Austria).

Cell apoptosis. Flow cytometry was used to analyze cell apoptosis and Annexin V-propidium iodide (AV-PI) staining was performed. Briefly, after treatment cells were harvested and washed three times with PBS. Following centrifugation for $10 \mathrm{~min}$, cells were resuspended in $500 \mu \mathrm{l}$ of binding buffer including $5 \mu \mathrm{l}$ FITC-conjugated Annexin V, the mixture was incubated in the dark for $10 \mathrm{~min}$, and then $5 \mu \mathrm{l}$ of PI was added. Ultimately, all specimens were assessed by flow cytometry with a FACSCalibur using CellQuest software (BD Biosciences, San Jose, CA, USA), and all the results are shown as a percentage of total cells, to quantitatively evaluate the rate of apoptosis.

Caspase- 9 activity. The caspase- 9 activity was assayed using the Caspase-9 Assay kit (Abcam), according to the manufacture's instruction. The fresh protein lysates from cells were prepared using cell lysis buffer. Then, $85 \mu \mathrm{l}$ of reaction buffer and $5 \mu \mathrm{l}$ of LEHD-pNA (Leu-Glu-His-Asp-p-nitroanilide) were added to each sample and incubated at $37^{\circ} \mathrm{C}$ for $2 \mathrm{~h}$. The absorbance was measured in an ELISA reader (Labsystems, Helsinki, Finland) at $405 \mathrm{~nm}$.

Statistical analysis. The SPSS version 19.0 software (SPSS, Inc., Chicago, IL, USA) was used to analyze the related data with $\chi^{2}$ or t-tests. The results were considered to indicate a statistically significant result at $\mathrm{P}<0.05$.

\section{Results}

HAX-1 is highly expressed in human prostate cancer cell lines. To investigate the expression of HAX-1 in human prostate cancer cells, we used RT-PCR to detect HAX-1 mRNA levels and western blotting to analyze the protein levels in normal human primary prostate epithelial cells, and the prostate cancer cell lines PC3, VCaP and DU145. RT-PCR analysis showed that the mRNA levels of HAX-1 in the prostate cancer cell lines were higher than that in the normal cells (Fig. 1A). Consistent with the results of mRNA levels, the protein levels in different prostate cancer cells were higher than that in the normal cells (Fig. 1B). It is noteworthy, that both the mRNA and protein levels of HAX-1 in DU145 were higher than those in $\mathrm{PC} 3$ and $\mathrm{VCaP}$.

Expression levels of HAX-1 in HAX-1 knockdown DU145 cells. On the basis that the expression of HAX-1 is higher in the DU145 cells than the other two prostate cancer cell lines, PC3 and VCaP, we generated HAX-1 knockdown DU145 cells by HAX-1 siRNA to investigate the function of HAX-1 in prostate 
A

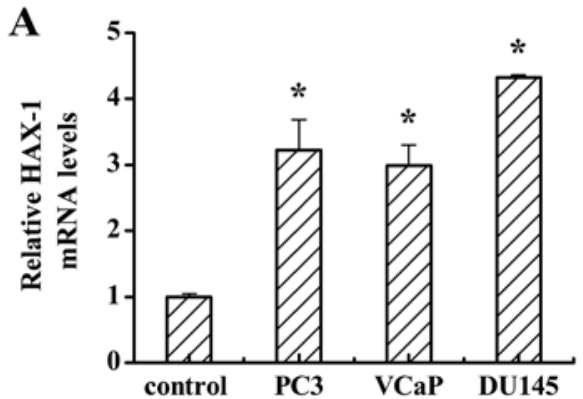

B

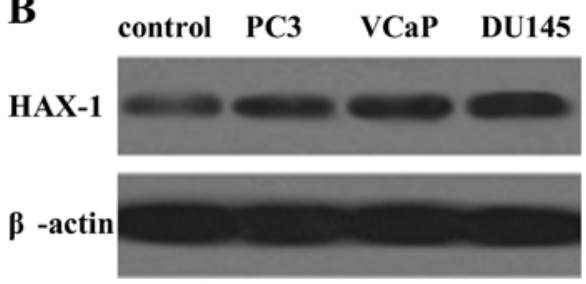

Figure 1. mRNA and protein levels of HAX-1 in control (normal human primary prostate epithelial cells) and three prostate cancer cell lines, $\mathrm{PC} 3, \mathrm{VCaP}$ and DU145 cell lines. (A) Relative HAX-1 mRNA levels in the cells. "P<0.05 compared with control. (B) HAX-1 protein levels in the cells. HAX-1, HS1 associated protein $\mathrm{X}-1$.

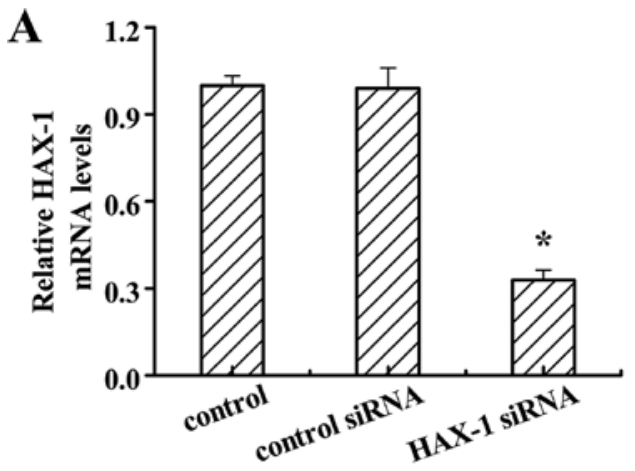

B

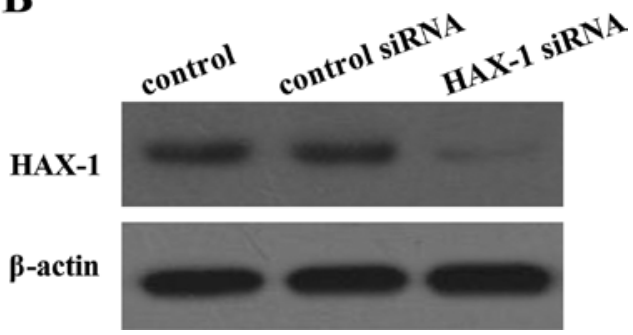

Figure 2. mRNA and protein levels of HAX-1 in DU145. (A) Relative HAX-1 mRNA levels in control cells (DU145 without any treatment), cells treated with control siRNA and HAX-1 siRNA, ${ }^{*} \mathrm{P}<0.05$ compared with control. (B) HAX-1 protein levels in control, control siRNA and HAX-1 siRNA-treated cells. HAX-1, HS1 associated protein X-1.

cancer cells. After HAX-1 siRNA treatment, the mRNA and protein levels of HAX-1 were significantly decreased (Fig. 2). These results confirmed that the HAX-1 knockdown-DU145 cells were successfully established.

Effect of HAX-1 knockdown on cell proliferation. Cell proliferation was assessed in DU145 cells after HAX-1 siRNA treatment by the MTT analysis. The results revealed that
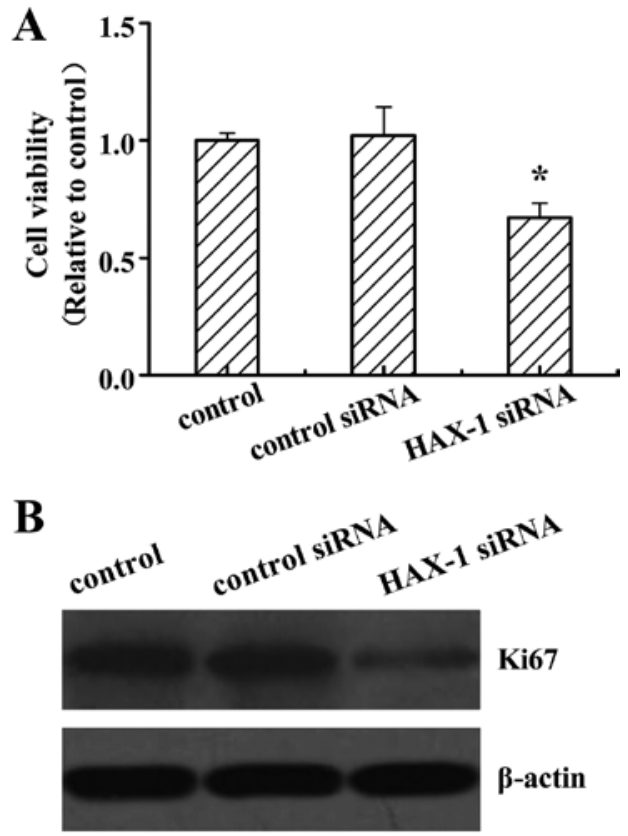

Figure 3. Effect of HAX-1 knockdown on DU145 viability. (A) Relative MTT absorbance in control cells (DU145 without any treatment), cells treated with control siRNA and HAX-1 siRNA, ${ }^{*} \mathrm{P}<0.05$ compared with control. (B) Ki67 protein levels in control cells (DU145 without any treatment), cells treated with control siRNA and HAX-1 siRNA, "P<0.05 compared with control. HAX-1, HS1 associated protein X-1.

HAX-1 knockdown significantly decreased the cell viability compared to the other two groups $(\mathrm{P}<0.05$; Fig. $3 \mathrm{~A})$. No differences were detected between the control and control siRNA-treated cells. Ki67 has been used as a marker for cell proliferation in cancer. We further analyzed the Ki67 protein expression levels, and revealed that HAX-1 siRNA suppressed the Ki67 protein expression (Fig. 3B).

Effect of HAX-1 knockdown on cell apoptosis. We next investigated the role of HAX-1 on DU145 apoptosis, after HAX-1 siRNA treatment. As shown in Fig. 4, TRAF4 knockdown notably increased cell apoptosis from 13 to $56 \%$ when compared to the control groups. No difference was found between the control and control siRNA groups $(\mathrm{P}<0.05$; Fig. 4A and B). Moreover, HAX-1 siRNA also induced the pro-apoptosis protein Bax, whereas inhibited the anti-apoptosis protein $\mathrm{Bcl}-2$ expression (Fig. 4C). These data reflected that HAX-1 is an anti-apoptosis factor in prostate cancer.

HAX-1 knockdown enhances viability and inhibits apoptosis in prostate cancer cells by caspase-9 inhibition. Caspase- 9 is a critical regulator of mitochondria-mediated apoptosis, and HAX-1 is one of the molecules that interacts with caspase-9 (11). To determine whether HAX-1 interacts with caspase- 9 in the progress of prostate cancer viability and apoptosis, the activity of caspase- 9 was determined in DU145 cells after HAX-1 knockdown. The results showed that HAX-1 knockdown markedly increased caspase- 9 activity $(\mathrm{P}<0.05$; Fig. 5A). The results of western blotting showed that HAX-1 knockdown promoted caspase-9 processing in DU145 cells. These results indicated that HAX-1 is a negative regulator of caspase-9 activation. 
A

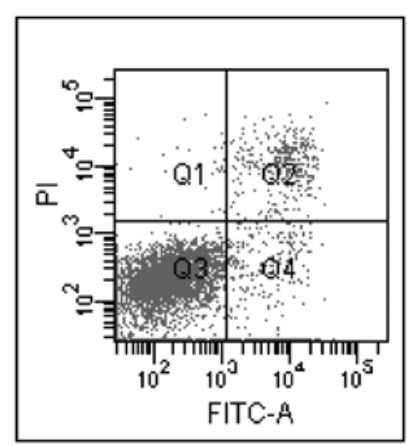

B

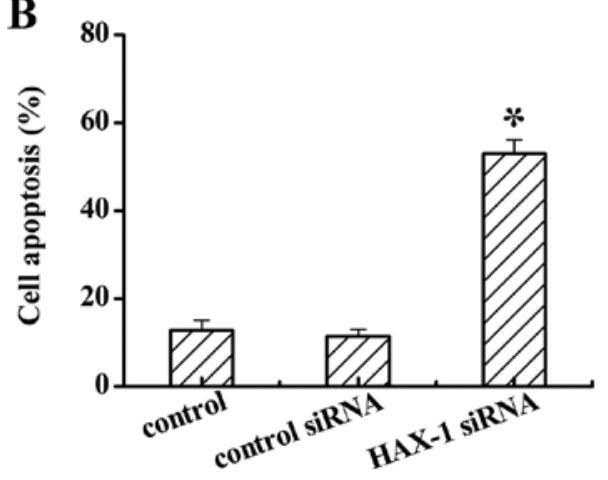

control siRNA

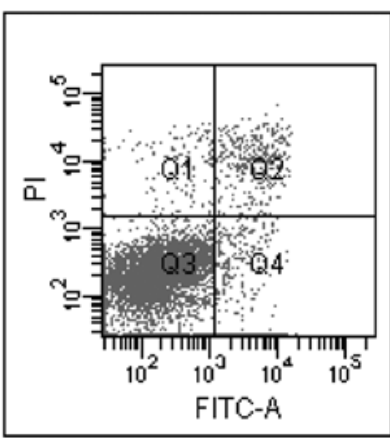

C

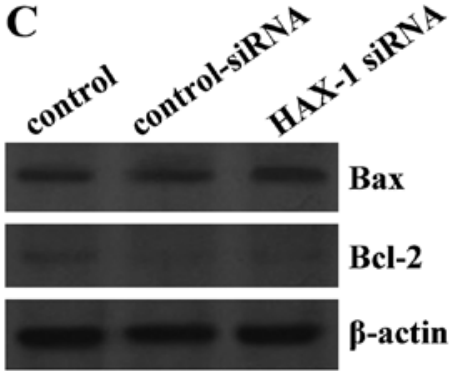

Figure 4. Effect of HAX-1 knockdown on DU145 apoptosis. (A) The effect of HAX-1 knockdown on cell apoptosis, cells were stained with Annexin V/PI. (B) Quantification of the data of B. * $\mathrm{P}<0.05$ compared with control. (C) Western blot analysis of the protein levels of Bcl-2 and Bax.
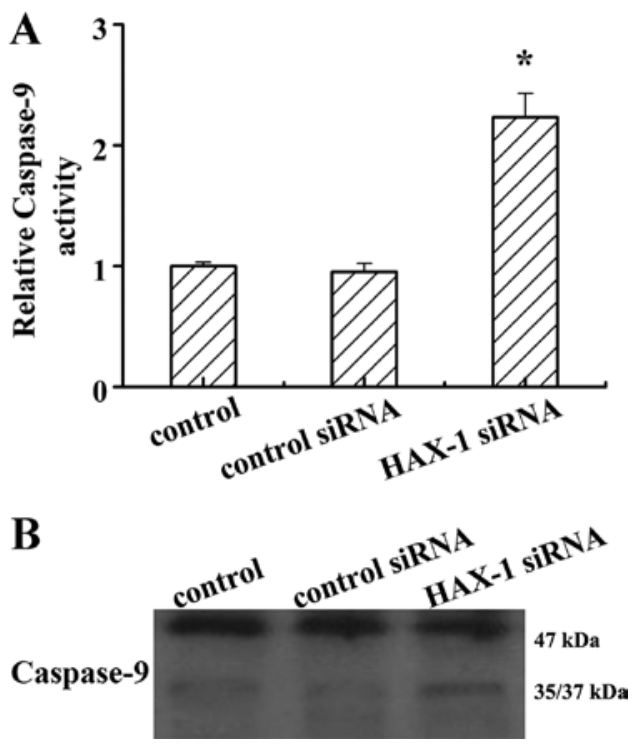

Figure 5. Effect of HAX-1 knockdown on caspase-9 activation in DU145. (A) Caspase-9 activity was measured after the cells were treated with control siRNA and HAX-1 siRNA, ${ }^{*} \mathrm{P}<0.05$ compared with control (DU145 without any treatment). (B) Caspase- 9 processing was determined after the cells were treated with control siRNA and HAX-1 siRNA.

HAX-1 enhances prostate cancer cell viability and apoptosis through inhibit caspase-9 activation. To further explore the function of caspase-9 signaling in HAX-1-induced prostate cancer viability and apoptosis, caspase-9, HAX-1 and HAX-1 siRNA were co-transfected to DU145 cells. As shown in Fig. 6A, cell viability was significantly increased in caspase-9 and HAX-1 co-transfected cells compared to caspase-9 only
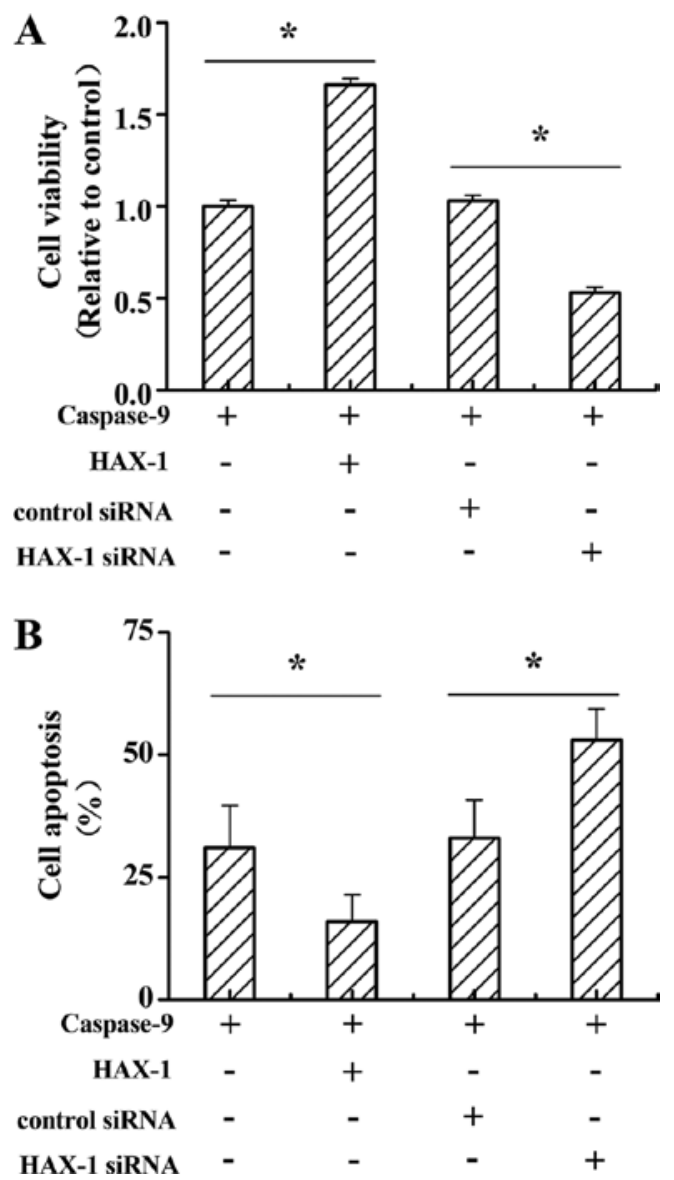

Figure 6. HAX-1 enhances DU145 viability and reduces apoptosis through the caspase-9 inactivation. (A) Cell viability and (B) apoptosis were detected after co-transfection with caspase- 9 and HAX-1, and HAX-1 siRNA. ${ }^{\mathrm{P}}<0.05$, comparison between two groups under the bar. 
transfected cells. Moreover, the cell viability in caspase- 9 and HAX-1 siRNA co-transfected cells was sharply decreased when compared to caspase- 9 and control siRNA co-transfected cells $(\mathrm{P}<0.05)$. The cell apoptosis in caspase-9 and HAX-1 co-transfected cells was markedly decreased when compared to the caspase- 9 only transfected cells. Furthermore, cell apoptosis in caspase- 9 and HAX-1 siRNA co-transfected cells was notably increased when compared to the caspase- 9 and control siRNA co-transfected cells $(\mathrm{P}<0.05)$.

\section{Discussion}

Induction of differentiation and apoptosis in cancer cells is a critical approach in cancer therapy (12). Substantial research has focused on the induction of apoptosis in prostate cancer. For example, Hsieh and $\mathrm{Wu}$ showed that resveratrol treatment is able to induce apoptosis in prostate cancer (13), and Hsu et al showed that the cyclooxygenase-2 inhibitor celecoxib induces apoptosis in human prostate cancer cells by blocking Akt activation (14). In fact, the regulation of apoptosis relies on multiple cell signaling mechanisms, cancer cells can employ a number of different strategies to suppress a protective apoptotic response, contributing to cancer development by promoting cell survival and resistance to antineoplastic drugs (15-17). In this regard, new anticancer therapeutics are needed to focus on the induction of cancer cell apoptosis through activation of the apoptotic pathway (18).

HAX-1 is a family of apoptotic regulators in disease including cancer (19-21). It has been reported that HAX-1 is highly expressed in different human melanoma cell lines (22). In this research, we first investigated that HAX-1 is highly expressed in prostate cancer cells, and further analysis found that HAX-1 knockdown sharply decreased prostate cancer cell proliferation and the expression of cell proliferating marker, Ki67. Moreover, HAX-1 knockdown significantly promoted apoptosis, enhanced the pro-apoptosis protein Bax, and inhibited the anti-apoptosis protein $\mathrm{Bcl}-2$ expression in prostate cancer. These data indicate that HAX-1 is an anti-apoptosis molecule in prostate cancer. Cell apoptosis is a complex process regulated by several molecules that function as either promoters, including Bax, Bak and caspases, or inhibitors of the cell death process such as Bcl-2, Bcl-xL and the IAPs (23-27). In addition, HAX-1 has been shown to interact with a number of cellular and viral proteins, thus confirming its involvement in multiple signaling pathways and cellular processes $(28,29)$.

In recent years, many cellular factors involved in apoptosis have been identified and their roles in the apoptotic pathway has been elucidated (30). Mitochondria is a key participant in cell death, and apoptotic cell death is characterized by a host of morphological and biochemical features, mitochondrial outer membrane permeabilization (MOMP) and the release of pro-apoptotic proteins (31). Activation of the BCL-2 family members Bax and Bak results in MOMP, and release of the pro-apoptotic proteins, such as cytochrome $c$ from the inter-membrane space into the cytosol. Cytochrome $c$ binds Apaf-1 forming the apoptosome and activating caspase- 9 . Once active, caspase-9 directly cleaves and activates caspase- 3 and caspase-7 (32-34). It has been found that HAX-1 directly binds to caspase-9, leading to post-mitochondrial inactivation of caspase-9, thus blocking the caspase-9-mediated cell apoptosis pathway in cardiac myocytes (9). It is noteworthy that we found, that HAX-1 knockdown enhanced caspase-9 activity, further experiments demonstrated that prostate cancer cell co-transfected with HAX-1 and caspase-9 promoted cell viability and reduced cell apoptosis. In contract, co-transfection of caspase- 9 and HAX-1 siRNA suppressed cell viability and enhanced apoptosis. This phenomenon, provides evidence that the HAX-1, inhibits cell apoptosis through caspase-9 inactivation.

In summary, in the present study we propose a new mechanism to account for apoptosis in prostate cancer by HAX-1 inhibition. A better understanding of this process could lead to the development of novel therapeutic strategies aimed at reducing tumor growth and the development of prostate cancer.

\section{References}

1. Varambally S, Dhanasekaran SM, Zhou M, Barrette TR, Kumar-Sinha C, Sanda MG, Ghosh D, Pienta KJ, Sewalt RG, Otte AP, et al: The polycomb group protein EZH2 is involved in progression of prostate cancer. Nature 419: 624-629, 2002.

2. Tsai HT, Penson DF, Makambi KH, Lynch JH, Van Den Eeden SK and Potosky AL: Efficacy of intermittent androgen deprivation therapy vs conventional continuous androgen deprivation therapy for advanced prostate cancer: A meta-analysis. Urology 82: 327-333, 2013.

3. Suzuki Y, Demoliere C, Kitamura D, Takeshita H, Deuschle U and Watanabe T: HAX-1, a novel intracellular protein, localized on mitochondria, directly associates with HS1, a substrate of Src family tyrosine kinases. J Immunol 158: 2736-2744, 1997.

4. Mirmohammadsadegh A, Tartler U, Michel G, Baer A, Walz M, Wolf R, Ruzicka T and Hengge UR: HAX-1, identified by differential display reverse transcription polymerase chain reaction, is overexpressed in lesional psoriasis. J Invest Dermatol 120: 1045-1051, 2003.

5. Sharp TV, Wang H-W, Koumi A, Hollyman D, Endo Y, Ye H, Du MQ and Boshoff C: K15 protein of Kaposi's sarcoma-associated herpesvirus is latently expressed and binds to HAX-1, a protein with antiapoptotic function. J Virol 76: 802-816, 2002.

6. Trebinska A, Rembiszewska A, Ciosek K, Ptaszynski K, Rowinski S, Kupryjanczyk J, Siedlecki JA and Grzybowska EA: HAX-1 overexpression, splicing and cellular localization in tumors. BMC Cancer 10: 76, 2010.

7. Jing YY, Li XL, Shi Q, Wang ZY, Guo Y, Pan MM, Tian C, Zhu SY, Chen C, Gong HS, et al: A novel PrP partner HS-1 associated protein X-1 (HAX-1) protected the cultured cells against the challenge of $\mathrm{H}_{2} \mathrm{O}_{2}$. J Mol Neurosci 45: 216-228, 2011.

8. Wei XJ, Li SY, Yu B, Chen G, Du JF and Cai HY: Expression of HAX-1 in human colorectal cancer and its clinical significance. Tumour Biol 35: 1411-1415, 2014.

9. Shaw J and Kirshenbaum LA: HAX-1 represses postmitochondrial caspase-9 activation and cell death during hypoxia-reoxygenation. Circ Res 99: 336-338, 2006.

10. Mahdavi M, Davoodi J, Zali MR and Foroumadi A: Concomitant activation of caspase-9 and down-regulation of IAP proteins as a mechanism of apoptotic death in HepG2, T47D and HCT-116 cells upon exposure to a derivative from 4-aryl-4H-chromenes family. Biomed Pharmacother 65: 175-182, 2011.

11. Han Y, Chen YS, Liu Z, Bodyak N, Rigor D, Bisping E, Pu WT and Kang PM: Overexpression of $\mathrm{HAX}-1$ protects cardiac myocytes from apoptosis through caspase-9 inhibition. Circ Res 99: 415-423, 2006.

12. Elstner E, Müller C, Koshizuka K, Williamson EA, Park D, Asou H, Shintaku P, Said JW, Heber D and Koeffler HP: Ligands for peroxisome proliferator-activated receptorgamma and retinoic acid receptor inhibit growth and induce apoptosis of human breast cancer cells in vitro and in BNX mice. Proc Natl Acad Sci USA 95: 8806-8811, 1998.

13. Hsieh TC and Wu JM: Differential effects on growth, cell cycle arrest, and induction of apoptosis by resveratrol in human prostate cancer cell lines. Exp Cell Res 249: 109-115, 1999.

14. Hsu AL, Ching TT, Wang DS, Song X, Rangnekar VM and Chen CS: The cyclooxygenase-2 inhibitor celecoxib induces apoptosis by blocking Akt activation in human prostate cancer cells independently of Bcl-2. J Biol Chem 275: 11397-11403, 2000. 
15. Fulda S: Evasion of apoptosis as a cellular stress response in cancer. Int J Cell Biol 2010: 370835, 2010.

16. Plati J, Bucur O and Khosravi-Far R: Apoptotic cell signaling in cancer progression and therapy. Integr Biol Camb 3: 279-296, 2011.

17. Akgul C: Mcl-1 is a potential therapeutic target in multiple types of cancer. Cell Mol Life Sci 66: 1326-1336, 2009.

18. Vidya Priyadarsini R, Senthil Murugan R, Maitreyi S, Ramalingam K, Karunagaran D and Nagini S: The flavonoid quercetin induces cell cycle arrest and mitochondria-mediated apoptosis in human cervical cancer (HeLa) cells through p53 induction and NF- $\mathrm{KB}$ inhibition. Eur J Pharmacol 649: 84-91, 2010.

19. Li WB, Feng J, Geng SM, Zhang PY, Yan XN, Hu G, Zhang CQ and Shi BJ: Induction of apoptosis by Hax-1 siRNA in melanoma cells. Cell Biol Int 33: 548-554, 2009.

20. Yap SV, Koontz JM and Kontrogianni-Konstantopoulos A: HAX-1: A family of apoptotic regulators in health and disease. J Cell Physiol 226: 2752-2761, 2011.

21. Li M, Tang Y, Zang W, Xuan X, Wang N, Ma Y, Wang Y, Dong Z and Zhao G: Analysis of HAX-1 gene expression in esophageal squamous cell carcinoma. Diagn Pathol 8: 47, 2013.

22. Ferri KF and Kroemer G: Organelle-specific initiation of cell death pathways. Nat Cell Biol 3: E255-E263, 2001.

23. Philchenkov A: Caspases: Potential targets for regulating cell death. J Cell Mol Med 8: 432-444, 2004.

24. Yin C, Knudson CM, Korsmeyer SJ and Van Dyke T: Bax suppresses tumorigenesis and stimulates apoptosis in vivo. Nature 385: 637-640, 1997

25. Youle RJ and Strasser A: The BCL-2 protein family: Opposing activities that mediate cell death. Nat Rev Mol Cell Biol 9: 47-59, 2008.
26. Zong WX, Li C, Hatzivassiliou G, Lindsten T, Yu QC, Yuan J and Thompson CB: Bax and Bak can localize to the endoplasmic reticulum to initiate apoptosis. J Cell Biol 162: 59-69, 2003.

27. Deveraux QL and Reed JC: IAP family proteins - suppressors of apoptosis. Genes Dev 13: 239-252, 1999.

28. Fadeel B and Grzybowska E: HAX-1: A multifunctional protein with emerging roles in human disease. Biochim Biophys Acta 1790: 1139-1148, 2009.

29. Cilenti L, Soundarapandian MM, Kyriazis GA, Stratico V, Singh S, Gupta S, Bonventre JV, Alnemri ES and Zervos AS: Regulation of HAX-1 anti-apoptotic protein by Omi/HtrA2 protease during cell death. J Biol Chem 279: 50295-50301, 2004.

30. Gupta K, Thakur VS, Bhaskaran N, Nawab A, Babcook MA, Jackson MW and Gupta S: Green tea polyphenols induce p53-dependent and p53-independent apoptosis in prostate cancer cells through two distinct mechanisms. PLoS One 7: e52572, 2012.

31. Martinou JC and Youle RJ: Mitochondria in apoptosis: Bcl-2 family members and mitochondrial dynamics. Dev Cell 21: 92-101, 2011.

32. Brentnall M, Rodriguez-Menocal L, De Guevara RL, Cepero E and Boise LH: Caspase-9, caspase-3 and caspase-7 have distinct roles during intrinsic apoptosis. BMC Cell Biol 14: 32, 2013.

33. Li P, Nijhawan D, Budihardjo I, Srinivasula SM, Ahmad M, Alnemri ES and Wang X: Cytochrome $c$ and dATP-dependent formation of Apaf-1/caspase-9 complex initiates an apoptotic protease cascade. Cell 91: 479-489, 1997.

34. Srinivasula SM, Ahmad M, Fernandes-Alnemri T and Alnemri ES: Autoactivation of procaspase- 9 by Apaf-1-mediated oligomerization. Mol Cell 1: 949-957, 1998. 